\title{
Intermodulation Distortion in Coupled-Resonator Filters With Nonuniformly Distributed Nonlinear Properties-Use in HTS IMD Compensation
}

\author{
Jordi Mateu, Member, IEEE, James C. Booth, Carlos Collado, Member, IEEE, and \\ Juan M. O'Callaghan, Senior Member, IEEE
}

\begin{abstract}
We present a general procedure for calculating intermodulation distortion in coupled-resonator filters that allows one to predict the performance of a nonlinear filter as a function of the general nodal matrix defining the filter and the material parameters that cause the nonlinear behavior. It is valid for almost any type of nonlinear distributed effects, including those produced by high-temperature superconductors, nonlinear dielectrics such as ferroelectrics, or superconductor/ferroelectric bilayers, and it is valid when the spatial distribution of nonlinearities is not uniform. The procedure has been validated with experimental measurements in an eight-pole quasi-elliptic superconducting filter. Using this procedure, we have assessed a combination of materials with different types of nonlinear effects to partially or completely mitigate the filter's nonlinear response. This includes superconducting filters with a ferroelectric pre- or post-distorter stage or even with intermediate ferroelectric compensation stages.
\end{abstract}

Index Terms-Ferroelectrics, harmonic balance, intermodulation products, nonlinearities, nonlinear measurements, superconducting filters.

\section{INTRODUCTION}

$\mathbf{H}$ IGH-TEMPERATURE superconductor (HTS) filters are known to achieve superior performance with reduced insertion loss and high selectivity in very small planar topologies [1]. At present, most commercial efforts have concentrated on front-end pre-select bandpass filters for wireless applications, where the HTS filter improves the sensitivity and selectivity of the receiver, increasing the coverage and improving the quality of service [2]. In spite of this, there are still limitations that may exclude their use in many applications at microwave frequencies. In particular, their inherent nonlinear response, which produces intermodulation distortion (IMD), is a serious problem in communication systems [3]. Quantifying and reducing this degradation is crucial for spreading the application of HTS filters and also for improving their benefits.

\footnotetext{
Manuscript received August 9, 2006; revised November 21, 2006. This work was supported under the Fulbright Program, by the Spanish Ministry of Science and Technology, and by the Spanish Government (CICYT) under Grant MAT-2005-05656-C03 and under Contract RYC-2005-001125. This work was supported in part by the U.S. Government.

J. Mateu and J. C. Booth are with the National Institute of Standards and Technology, Boulder, CO 80305 USA (e-mail: jmateu@boulder.nist.gov; james.booth@boulder.nist.gov).

C. Collado and J. M. O'Callaghan are with the Department of Signal Theory and Communications, Universitat Politècnica de Catalunya, Barcelona 08034, Spain (e-mail: collado@tsc.upc.edu; joano@tsc.upc.edu).

Digital Object Identifier 10.1109/TMTT.2007.892802
}

Recent work discusses the possibility of combining superconducting thin films with nonlinear dielectrics (such as ferroelectrics) to partially or completely cancel the nonlinear effects in superconducting devices such as filters [4]. The cancellation conditions in [4] refer to the case of a nonlinear transmission line where the properties of the dielectric and conductor are uniform along the line (e.g., in a planar line with an HTS/ferroelectric bilayer). Since this could give rise to high loss due to the low loss tangent that most ferroelectrics have, in this paper, we explore alternative configurations for IMD cancellation where the nonlinear dielectric is placed only (or dominantly) in certain areas of the planar layout.

Additionally, this paper presents a procedure to calculate the IMD produced by the filter from its nodal matrix [5] and from the parameters of the distributed nonlinearities in its resonators. This procedure is applicable to almost any type of distributed nonlinear effects, including those produced by HTS films, ferroelectric layers in the substrate, or HTS/ferroelectric bilayers, and can also be applied to the cases referred above where IMD compensation is achieved with a distribution of nonlinearities that is not spatially homogeneous.

\section{NONUNIFORMLY DistribUted NONLINEAR PROPERTIES IN TRANSMISSION LINES AND Single RESONATORS}

Here, we extend the analysis made in [4] to transmission lines and transmission line resonators with nonlinearities that are not uniformly distributed along the line. As detailed in [4], [6]-[9], the equivalent circuit of a segment of transmission line with nonlinearities in the conductor and dielectric follows the equivalent circuit in Fig. 1(c). When dielectric nonlinearities are negligible or nonexisting, the equivalent circuit corresponds to that in Fig. 1(a). Similarly, Fig. 1(b) shows the equivalent circuit of a segment with negligible or nonexisting conductor nonlinearities.

In all the above cases, conductor nonlinearities could be produced by a superconducting material, whereas dielectric nonlinearities could come from a ferroelectric material.

Assuming a $p$ power law dependence of the circuit parameters in Fig. 1, we obtain

$$
\begin{aligned}
L(i) & =L_{0}+\Delta L(i) \text { with } \Delta L(i)=\Delta L_{p}|i|^{p} \\
R(i) & =R_{0}+\Delta R(i) \text { with } \Delta R(i)=\Delta R_{p}|i|^{p} \\
C(v) & =C_{0}+\Delta C(v) \text { with } \Delta C(v)=\Delta C_{p}|v|^{p} \\
G(v) & =G_{0}+\Delta G(v) \text { with } \Delta G(v)=\Delta G_{p}|v|^{p}
\end{aligned}
$$




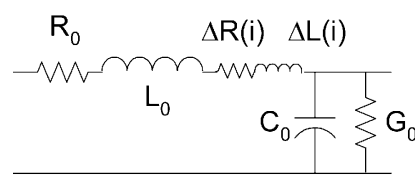

a)

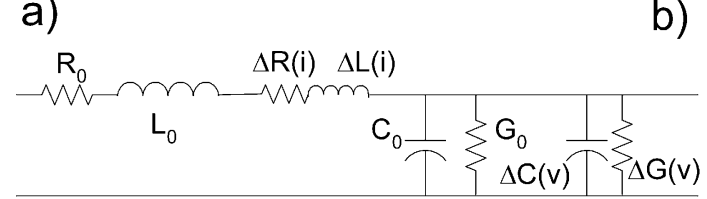

c)

Fig. 1. Equivalent circuit of a transmission line elemental segment of length $d z$ with: (a) nonlinear effects in the conductor part, (b) nonlinear effects in the dielectric, and (c) nonlinear effects in both the conductor and dielectric parts. Note that formally the circuit parameters should be scaled by $d z$.

where $L_{0}(\mathrm{H} / \mathrm{m}), R_{0}(\Omega \mathrm{m}), C_{0}(\mathrm{~F} / \mathrm{m})$, and $G_{0}(\mathrm{~S} / \mathrm{m})$ are the linear distributed parameters and $\Delta L_{p}\left(\mathrm{H} / \mathrm{m} \cdot i^{p}\right), \Delta R_{p}\left(\Omega \mathrm{m} \cdot i^{p}\right)$, $\Delta C_{p}\left(\mathrm{~F} / \mathrm{m} \cdot v^{p}\right)$, and $\Delta G_{p}\left(\mathrm{~S} / \mathrm{m} \cdot v^{p}\right)$ are the scaling factors setting the strength of the nonlinear effects.

Although the procedure described henceforth might be applied for any power dependence, we will use a quadratic nonlinear dependence $p=2$, which agrees with many results found in the literature for both HTS [10], [11] and ferroelectric thin films [7]-[9]. Details on how to apply this procedure for a given type of power dependence $p$ in superconductors may be found in [12].

\section{A. IMD in Transmission Lines With Nonuniformly Distributed Nonlinear Properties}

We first consider the case of a length of nonlinear transmission line with negligible (or nonexisting) dielectric nonlinearities. The IMD current $I_{12, H}$ produced at frequency $2 f_{1}-f_{2}$ at the output of an HTS transmission line segment of length $l_{H}$ can be calculated from [4, eq. (25)] as follows:

$$
I_{12, H}=-\frac{3}{8} \frac{I_{1}^{2} I_{2}^{*}}{Z_{0, H}} A_{H, 12} l_{H} e^{-\gamma_{H} l_{H}}
$$

where $I_{1}$ and $I_{2}$ are the currents at fundamental frequencies $f_{1}$ and $f_{2}$ feeding the line, $\gamma_{H}$ is the propagation constant of the HTS line, $Z_{0, H}$ is the characteristic impedance of the superconducting line, and the term $A_{H, 12}=\Delta R_{2}+j \omega_{12} \Delta L_{2}$ accounts for the nonlinear effects [6], where $\omega_{12}=2 \omega_{1}-\omega_{2}$.

Reference [4, eq. (25)] also gives the condition for considering dielectric nonlinearities negligible: $A_{H, 12} \gg$ $A_{F, 12} Z_{0}^{2}\left|Z_{0}\right|^{2}$, where the term $A_{F, 12}=\Delta G_{2}+j \omega_{12} \Delta C_{2}$ accounts for the nonlinearities in the dielectric.

Repeating the analysis above for a low-loss transmission line with dominant nonlinearities in the dielectric, we may use [4, eq. (25)] to write

$$
I_{12, F}=-\frac{3}{8} I_{1}^{2} I_{2}^{*} Z_{0, F}\left|Z_{0, F}\right|^{2} A_{F, 12} l_{F} e^{-\gamma_{F} l_{F}}
$$

where $\gamma_{F}$ is the propagation constant of the line, $l_{F}$ is its length, and $Z_{0, F}$ is its characteristic impedance. Similarly to the previous case, dielectric nonlinearities might be considered dominant if $A_{F, 12} Z_{0}^{2}\left|Z_{0}\right|^{2} \gg A_{H, 12}$.

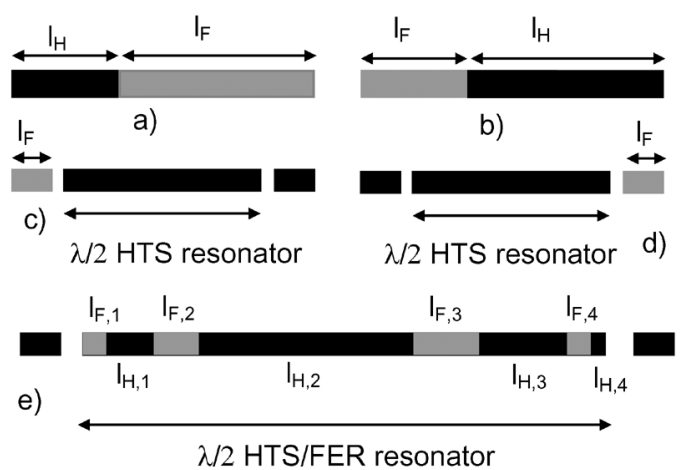

Fig. 2. Outline of the structures analyzed in Section II. (a) HTS (input) and ferroelectric (output) cascaded transmission line segments. (b) Ferroelectric (input) and HTS (output) cascaded transmission line segments. (c) HTS resonator with ferroelectric input feeding line. (d) HTS resonator with ferroelectric output feeding line. (e) HTS resonator with interstage ferroelectric segments. $l_{H}$ and $l_{F}$ are the length of the superconducting and ferroelectric segments, respectively.

Below we calculate the IMD resulting from cascading two nonlinear transmission lines corresponding to the two cases above [see Fig. 2(a) and (b)] and find the conditions for IMD cancellation at the output. We assume perfect impedance matching between them $\left(Z_{0, H}=Z_{0, F}=Z_{0}\right)$.

1) HTS + Ferroelectric Transmission Line: As shown in Fig. 2(a), this case considers a traveling wave that goes from a transmission line whose nonlinearities are dominated by the HTS to one where the nonlinearities are dominated by a ferroelectric. By using (5) and (6) and considering the propagation of the IMD and fundamental signals along the structure of Fig. 2(a), the resulting IMD is

$$
\begin{gathered}
I_{12, H F}=-\frac{3}{8} \frac{I_{1}^{2} I_{2}^{*}}{Z_{0}}\left[A_{H, 12} l_{H}+Z_{0}^{2}\left|Z_{0}\right|^{2} A_{F, 12} l_{F} e^{-2 \alpha_{H} l_{H}}\right] \\
\cdot e^{-\gamma_{H} l_{H}} e^{-\gamma_{F} l_{F}}
\end{gathered}
$$

where $Z_{0}$ is the characteristic impedance of the line and $\alpha_{H}$ is the real part of $\gamma_{H}$.

Since the nonlinear effects for the HTS and ferroelectric are in opposite phase [8], [13], we find the condition for IMD cancellation as

$$
A_{H, 12} l_{H}=-Z_{0}^{2}\left|Z_{0}\right|^{2} A_{F, 12} l_{F} e^{-2 \alpha_{H} l_{H}} .
$$

2) Ferroelectric + HTS Transmission Line: As shown in Fig. 2(b), this case considers a traveling wave that goes from a transmission line whose nonlinearities are dominated by the ferroelectric to one where the nonlinearities are dominated by the HTS. In analogy to the previous case, the IMD for this structure results as follows:

$$
\begin{array}{r}
I_{12, F H}=-\frac{3}{8} \frac{I_{1}^{2} I_{2}^{*}}{Z_{0}}\left[A_{H, 12} l_{H} e^{-2 \alpha_{F} l_{F}}+\right. \\
\left.+Z_{0}^{2}\left|Z_{0}\right|^{2} A_{F, 12} l_{F}\right] \\
\cdot e^{-\gamma_{H} l_{H}} e^{-\gamma_{F} l_{F}}
\end{array}
$$

where $\alpha_{F}$ is the real part of $\gamma_{F}$.

The cancellation condition is now

$$
A_{H, 12} l_{H} e^{-2 \alpha_{F} l_{F}}=-Z_{0}^{2}\left|Z_{0}\right|^{2} A_{F, 12} l_{F} .
$$


For low losses or very short input lines, both conditions, (8) and (10) are almost identical.

Note that the main difference when compared to the results presented in [4] is that the length of the lines may be used as a fitting parameter to cancel the nonlinear effects without needing to modify the nonlinear terms $A_{H, 12}$ and $A_{F, 12}$. Also note that this would have an important impact on practical devices since we can adjust the nonlinear response just by changing the length of the lines.

\section{B. IMD in a Single Resonator With Nonuniformly Distributed Nonlinear Properties}

For a resonator, we assess three different cases of combining HTS and ferroelectric segments, respectively, illustrated in Fig. 2(c)-(e). For analyzing these cases, we also use the results reported in [4] and [6]. In [4], we obtained the output IMD current for a general bilayer HTS/ferroelectric resonator as

$$
I_{12}=-\frac{9}{16} \frac{Q_{L}}{L_{d} \omega_{12}} I_{1}^{2} I_{2}^{*}\left[A_{H, 12}+Z_{0}^{2}\left|Z_{0}\right|^{2} A_{F, 12}\right]
$$

where $Q_{L}$ is the loaded quality factor of the resonator and $L_{d}$ is the distributed inductance of the half-wave transmission line resonator. Equation (11) assumes a sinusoidal distribution of the current along the resonator [4]. Note that for purely HTS nonlinear effects (cases 1 and 2 below), $A_{F, 12}=0$.

1) Half-Wave HTS Transmission Line Resonator With a Ferroelectric Input Feeding Line: Fig. 2(c) illustrates this case. The following equation gives the IMD resulting in this case:

$$
\begin{aligned}
I_{12}=-\frac{3}{8} I_{1}^{2} I_{2}^{*}\left[Z_{0}\left|Z_{0}\right|^{2} A_{F, 12} l_{F}\left(1-\frac{Q_{L}}{Q_{0}}\right)\right. \\
\left.+\frac{3}{2} \frac{Q_{L}}{L_{d} \omega_{12}} A_{H, 12} e^{-2 \alpha_{F} l_{F}}\right] e^{-\gamma_{F} l_{F}}
\end{aligned}
$$

where $Q_{0}$ is the unloaded quality factor of the resonator. Note that the term considering the IMD at the output of the resonator due to the ferroelectric input feeding line (6) is scaled by the transfer coefficient of the resonator $\left(1-\left(Q_{L} / Q_{0}\right)\right)$ [14], whereas the IMD generated by the HTS resonator (11) is modified by the propagation of the fundamental tones ( $I_{1}$ and $I_{2}$ ) along the ferroelectric feeding line. From (12), we obtain the cancellation condition as

$$
\frac{3}{2} \frac{Q_{L}}{\omega_{12} L_{d}} A_{H, 12}=-Z_{0}\left|Z_{0}\right|^{2} A_{F, 12} l_{F}\left(1-\frac{Q_{L}}{Q_{0}}\right) .
$$

2) Half-Wave HTS Transmission Line Resonator With a Ferroelectric Output Feeding Line: Fig. 2(d) shows the diagram defining this case. As in the case above, we obtained the output IMD current from (6) and (11) as follows:

$$
\begin{aligned}
I_{12}=-\frac{3}{8} I_{1}^{2} I_{2}^{*}\left[Z_{0}^{2}\left|Z_{0}\right|^{2} A_{F, 12}\right. & l_{F}\left(1-\frac{Q_{L}}{Q_{0}}\right)^{3} \\
& \left.+\frac{3}{2} \frac{Q_{L}}{L_{d} \omega_{12}} A_{H, 12}\right] e^{-\gamma_{F} l_{F}}
\end{aligned}
$$

where now the ferroelectric segment is fed by the fundamental currents $I_{1}$ and $I_{2}$ affected by the transfer coefficient of the res- onator. By using (14), the following cancellation condition results:

$$
\frac{3}{2} \frac{Q_{L}}{\omega_{12} L_{d}} A_{H, 12}=-Z_{0}\left|Z_{0}\right|^{2} A_{F, 12} l_{F}\left(1-\frac{Q_{L}}{Q_{0}}\right)^{3} .
$$

Equations (14) and (15) are very significant because they show us how the nonlinear effects in an HTS resonator may be cancelled by pre-distorting the signals feeding the resonator or by post-distorting the signals at the output of the resonator without altering its performance. As in Section II-A, the cancellation conditions in (14) and (15) may be controlled by tuning the length of the ferroelectric feeding lines. Note, however, that for input tones that are not at the center of the resonance, the cancellation conditions are not valid because they assume that peak currents (i.e., those of the IMD and fundamentals) are those obtained at resonance. Therefore, the output IMD currents in these two cases have strong frequency dependence, albeit they are minimum at the resonant frequency.

3) Intermediate Compensation Stages: This case corresponds to the one illustrated in Fig. 2(e) and avoids the frequency dependence mentioned above by introducing segments with dominant dielectric nonlinearities in the resonator. As in the bilayer HTS/ferroelectric case (11), we use the procedure described in [4] and [6], and consider the fact that the circuit distributed nonlinear parameters are not uniform. The resulting IMD is

$I_{12}=-\frac{3}{4} \frac{I_{1}^{2} I_{2}^{*} Q_{L}}{L_{d, H} \Gamma_{H}+L_{d, F} \Gamma_{F}}\left[Z_{0}\left|Z_{0}\right|^{2} A_{F, 12} \Gamma_{F}^{\prime}+A_{H, 12} \Gamma_{H}^{\prime}\right]$

where the geometrical factors are $\Gamma_{F}=\int_{l_{F}} g(z)^{2} d z, \Gamma_{H}=$ $\int_{l_{H}} g(z)^{2} d z, \Gamma_{F}^{\prime}=\int_{l_{F}} g(z)^{4} d z$, and $\Gamma_{H}^{\prime}=\int_{L_{H}} g(z)^{4} d z$, where $g(z)=\sin (\pi z / l)$, and $l_{H}\left(=\left[l_{H, 1} l_{H, 2} l_{H, 3} l_{H, 4}\right]\right)$ and $l_{F}\left(=\left[l_{F, 1} l_{F, 2} l_{F, 3} l_{F, 4}\right]\right)$ are the intervals over which these geometrical factors are evaluated, respectively, defined by the position and length of the segments whose nonlinear effects are dominated by HTS and the dielectric, respectively. $L_{d, H}$ and $L_{d, F}$ are the distributed inductance corresponding to the $l_{H}$ and $l_{F}$ segments, respectively.

The cancellation condition in this case is

$$
A_{H, 12} \Gamma_{H}^{\prime}=-Z_{0}\left|Z_{0}\right|^{2} A_{F, 12} \Gamma_{F}^{\prime}
$$

Note that (16) is still valid when the conductor in the segments with dominant dielectric nonlinearities is an HTS. This eases the practical implementation of this structure. A possible embodiment of Fig. 2(e) could consist of a planar transmission line with a uniform HTS conductor and a nonuniform distribution of ferroelectrics along the line.

Likewise, (16) also holds for a resonator with only conductor nonlinearities $\left(\Gamma_{F}=\Gamma_{F}^{\prime}=0\right)$ so it will be used as a general equation regardless of whether the segments with dominant dielectric nonlinearities are used or not.

\section{IMD IN AN $N$-COUPLED-RESONATOR FILTER}

The analytical procedure for obtaining a closed-form expression for calculating the IMD in bandpass filters with nonuni- 


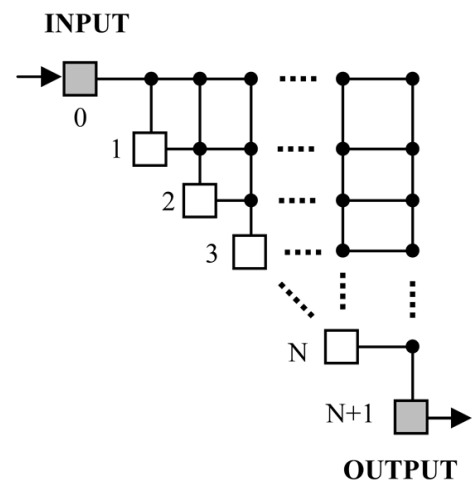

Fig. 3. Diagram of a general coupled-resonator filter topology. Square gray boxes represent the loads at the input and output ports, the square white boxes represent the resonators, and the dots represent the couplings.

formly distributed nonlinear effects is described here. This expression relates the IMD at the output of the filter to the nonlinear distributed effects by means of the coupling matrix of the filter. A different approach for calculating the intermodulation response in superconducting filters has been proposed in [15], accurately assessing the intermodulation effects in a communication system. However, [15] deals with neither the distributed nature of the nonlinear effects, nor their nonuniform distribution along the resonators forming the filter.

Fig. 3 outlines a general diagram for representing a wide range of coupled-resonator filter topologies [5]. In Fig. 3, the square gray boxes represent the loads at the input and output ports, the square white boxes represent the resonators, and the dots represent the couplings. To obtain the equivalent circuit of the general topology of Fig. 3, we may substitute the resonators by parallel $R L C$ equivalent circuits, the couplings by impedance or admittance inverters and the input and output ports by matched impedances $Z_{0}$ or admittances $Y_{0}$. Details on how to obtain this equivalent circuit as a function of the design parameters of the filter may be found in [5]. The equivalent circuit is then analyzed to obtain its impedance matrix $Z$, which is crucial for the analysis performed here.

To describe the procedure, this section has been organized in three subsections corresponding to the three main steps of the procedure. In the first step, we obtain the current of the fundamental signals at each resonator. From these currents, the second step describes how to obtain the currents of the IMDs generated at each resonator as a sum of the IMD generated in the resonator itself plus the IMD coupled from other resonators in the filter. Finally, the third step provides an expression for the total current of the IMDs coupled to the load. We then particularize this expression for HTS nonlinear distributed effects.

\section{A. Fundamental Signals on the Resonator}

The current of the fundamental signals coupled to each resonator affects the IMD generated along the filter [see (11), (12), (14), and (16)], thereby affecting the nonlinear filter performance. These currents depend on the coupling between resonators, their quality factors, and their resonant frequencies. All this information is included in the impedance matrix of the filter [5].
To evaluate the IMD of the filter, we feed the filter with two fundamental tones. We define these fundamental signals as two current sources at the input of the filter (see Fig. 3), $I_{s_{-} f 1}$ and $I_{s_{-} f 2}$, where $f_{1}$ and $f_{2}$ are the fundamental frequencies. Using the impedance matrix of the filter we determine the voltage at each resonator due to the fundamental currents [5]

$$
\begin{gathered}
{\left[\begin{array}{ccccc}
Z_{0,0} & Z_{0,1} & \cdots & Z_{0, N} & Z_{0, N+1} \\
Z_{1,0} & Z_{1,1} & \cdots & Z_{1, N} & Z_{1, N+1} \\
\vdots & \vdots & \ddots & \vdots & \vdots \\
Z_{N, 1} & Z_{N, 2} & \cdots & Z_{N, N} & Z_{N, N+1} \\
Z_{N+1,1} & Z_{N+1,2} & \cdots & Z_{N+1, N} & Z_{N+1, N+1}
\end{array}\right]\left[\begin{array}{c}
I_{s_{-} f_{j}} \\
0 \\
0 \\
\vdots \\
0
\end{array}\right]} \\
=\left[\begin{array}{c}
V_{0} \\
V_{1} \\
V_{2} \\
\vdots \\
V_{N+1}
\end{array}\right] .
\end{gathered}
$$

Note that the subscripts of the impedance matrix parameters go from 0 to $N+1$ according to the nodes of the equivalent circuit of Fig. 3, $V_{i}$ being the voltage at the $i$ th resonator (for $i$ from 1 to $N$ ).

The power dissipated by $V_{i}$ in the $i$ th resonator [16] is $P_{d_{i}}=$ $\left(\left|V_{i}\right|^{2} / R_{i}\right)$, where $R_{i}$ is the lumped resistance of its $R L C$ equivalent circuit. This power should be equal to the power dissipated by the current distribution along the distributed resonator

$$
P_{d_{i}}=\frac{1}{2} \frac{\omega\left[L_{d_{i}, F} \Gamma_{F_{i}}+L_{d_{i}, H} \Gamma_{H_{i}}\right]}{Q_{0 i}}
$$

where $Q_{0 i}$ is the unloaded quality factor of the $i$ th resonator. Typically, for half-wave resonators, $g_{i}(z)=\sin \left(\pi z / l_{i}\right)$, where the length of the resonator $l_{i}=\lambda / 2$. Therefore, the current distribution at the $i$ th resonator can be written as

$$
\begin{array}{r}
I_{i_{-} f_{j}}(z)=Z_{i, 0} \cdot I_{s_{-} f_{j}} \sqrt{\frac{4 Q_{0 i}}{\omega R_{i}\left(L_{d_{i}, F} \Gamma_{F}+L_{d_{i}, H} \Gamma_{H}\right)}} \\
\cdot \sin \left(\frac{\pi z}{l_{i}}\right) .
\end{array}
$$

Equations (19) and (20) can also be applied to other resonant modes, i.e., other $g_{i}(z)$. Note that the term $Z_{i, 0}$ also accounts for the frequency dependence of the incident signals coupled to the $i$ th resonator.

\section{B. IMD Currents at the Resonators}

Once we know the fundamental currents at each resonator, we may find the IMD at each resonator as a sum of the IMD currents coupled from other resonators plus the IMD current generated at that resonator. The latter can be calculated from (16) with a loaded quality factor of the $i$ th resonator obtained from the impedance matrix

$$
Q_{L i}=Q_{0 i} \frac{\operatorname{Re}\left[Z_{i, i}\right]}{R_{i}}
$$

where $Z_{i i}$ is the impedance seen from node $i$, which is real at resonance and depends on the $i$ th resonator and its coupling to 
all other resonators. For a half-wave resonator, $R_{i}$ is related to its unloaded quality factor as $Q_{0 i}=\left(R_{i} \pi / 2 Z_{0 i}\right), Z_{0 i}$ being the characteristic impedance of the resonant line [16].

To account for the IMD coupled in the $i$ th resonator from another $j$ th resonator, we define a transfer coefficient as

$$
k_{i j}=\frac{\operatorname{Re}\left[Z_{i, j}\right]}{\operatorname{Re}\left[Z_{j, j}\right]} .
$$

Note that this transfer coefficient times the total loaded quality factor (21) gives the external quality factor from the $j$ th resonator to the $i$ th resonator [14], [16]. By using (16) for each resonator and (20)-(22), we find the total IMD at the $i$ th resonator

$$
\begin{array}{r}
I_{\mathrm{IMD} i_{-} T}(z)=\sum_{j=1}^{N} T_{j} \gamma_{j} I_{j_{-f_{1}} I_{j_{-} f_{2}}^{*}}\left[Z_{0}\left|Z_{0}\right|^{2} A_{F, 12} \Gamma_{F}^{\prime}+A_{H, 12} \Gamma_{H}^{\prime}\right] \\
\cdot \frac{Q_{L j} k_{i j}}{\omega W_{0 j}} g_{i}(z)
\end{array}
$$

where, for quadratic nonlinear effects, $p=2$ in (1)-(4), $T=$ $3 / 4, \gamma=3 / 16$, and $W_{0, i}=\left[L_{d_{i}, H} \Gamma_{H}+L_{d_{i}, F} \Gamma_{F}\right] / 2$. Note that the transfer coefficient from the $i$ th to $i$ th resonator $k_{i i}=1$.

In conclusion, the IMD distribution in each resonator depends on the IMD generated at the resonator itself plus the contribution coupled from the IMD generated at other resonators. Each term of the addition (23) corresponds to the IMD contribution of each resonator, respectively. The phases of these terms depend on the phases of the fundamentals $\left(I_{j_{-} f_{1}}\right.$ and $\left.I_{j_{-} f_{2}}\right)$ on the resonators and the phases of the transfer coefficients $\left(k_{i j}\right)$. Note that their phases are defined by the impedance matrix of the filter. Although it might be possible to synthesize the phases of these terms, it could certainly affect the frequency response of the filter [5], hence, new filter synthesis procedures may be developed to satisfy the frequency response of the filter and obtain a reduction of the IMD. On the other hand, the phase and magnitude of each term of the addition also depends on the value of the nonlinear term $\left(Z_{0}\left|Z_{0}\right|^{2} A_{F, 12} \Gamma_{F}^{\prime}+A_{H, 12} \Gamma_{H}^{\prime}\right)$, thus a possibility exists to design the various resonators in a filter so that their values of $A_{F}$ and $A_{H}$ produce and overall cancellation of the IMD currents in the filter. Equation (23) may also be useful to find the resonators having the strongest contribution to the IMD output current, which do not necessarily have to be the ones with the highest fundamental currents since the IMD contribution is also affected by the couplings.

\section{IMD Coupled to the Load}

Although the current distribution and strength of the IMD along each resonator of the filter may be useful from a design point-of-view, what shows the impact of the nonlinear effects of the material on the nonlinear performance of the filter is the IMD at the output of the filter.

To obtain the IMD at the output of the filter, we again define a transfer coefficient to relate the IMD generated at each resonator to its contribution to the IMD at the load. As in (22), this coefficient may be obtained from the impedance matrix of the filter by

$$
k_{L j}=\frac{\operatorname{Re}\left[Z_{N+1, j}\right]}{\operatorname{Re}\left[Z_{j, j}\right]} .
$$

Doing so, we may define the IMD current at the output of the filter $\left(I_{\mathrm{IMD}_{L}}\right)$ as

$$
\begin{array}{r}
I_{\mathrm{IMD}_{L}}=\sum_{j=1}^{N} T_{j} \gamma_{j} I_{j_{-} f_{1}}^{2} I_{j_{-} f_{2}}^{*}\left[Z_{i 0}\left|Z_{i 0}\right|^{2} A_{F, 12} \Gamma_{F}^{\prime}+A_{H, 12} \Gamma_{H}^{\prime}\right] \\
\cdot \frac{Q_{L j} k_{L j}}{\omega W_{0 j}} \cdot
\end{array}
$$

Note that (25) is a particular case of (23) so that the earlier comments on the contribution of each resonator, and the effects for a better design of a filter based on its nonlinear performance (described in Section III-B), are also valid for (25). This expression is completely general and accounts for a possible nonhomogeneous spatial distribution of the nonlinear effects and the possibility of having different quality factors in the resonators forming the filter.

In an HTS bandpass filter made by coupling identical halfwave resonators with quadratic nonlinear effects, we may simplify (25) to obtain

$$
I_{\mathrm{IMD}_{L}}=\frac{9}{16} \frac{(\Delta R+j \omega \Delta L)}{\omega L_{d}} \sum_{j=1}^{N} I_{j_{-f_{1}}}^{2} I_{j_{-} f_{2}}^{*} Q_{L j} k_{L j}
$$

where the values of the fundamental signals at each resonator are defined by (20).

\section{CROSS-CHECK: IMD Simulations AND MEASUREMENTS}

The above procedure is checked here by assessing the resulting IMD in a simulated three-pole Chebyshev bandpass filter and in an eight-pole quasi-elliptic (QE) bandpass filter that has been fabricated and measured [17]. In the former case, we will have full knowledge of the impedance matrix and we will be able to compare the theoretical equations previously derived with circuit simulations based on harmonic balance [6], [18]. In the latter case, the impedance matrix is not fully known and has to be fitted to measurements, but once this is obtained, we will be able to compare measurements, theory, and simulations, albeit subject to the fitting error of the impedance matrix.

Harmonic-balance simulations are performed on equivalent circuits of the filters in which the transmission line resonators are modeled by a cascade of many elemental segments like the ones shown in Fig. 1. Depending on the transmission line segment that we are modeling, we chose between the elemental segment of Fig. 1(a), (b), or (c). Note that cascading elemental segments corresponding to HTS transmission lines, ferroelectric transmission lines, or bilayer HTS/ferroelectric transmission lines, we define a nonuniformly distributed nonlinear effects. Couplings between different resonators are simply modeled by an admittance inverter [17]. The resulting equivalent-circuit model is then analyzed with harmonic-balance techniques. This simulation technique has been extensively used to simulate 
TABLE I

IMD LOAD CURRENT IN HTS FILTER

\begin{tabular}{lcc}
\hline \hline & Mag (A) & Phase $\left(^{\circ}\right)$ \\
\hline $1^{\text {st }}$ Res. to the load & $4.419 \mathrm{e}-12$ & 89.4 \\
$2^{\text {on }}$ Res. to the load & $1.050 \mathrm{e}-11$ & 89.4 \\
$3^{\text {rd }}$ Res. to the load & $4.419 \mathrm{e}-12$ & 89.4 \\
Total & $\mathbf{8 . 9 4 3 e - 1 1}$ & 89.4 \\
\hline \hline
\end{tabular}

devices with nonlinear distributed effects [4], [6]. Several references show consistency between analytical formulation, simulation, and experiments.

\section{A. Three-Pole Chebyshev Filter}

The design parameters of the filter are $g_{0}=1, g_{1}=$ $0.6292, g_{2}=0.9703, g_{3}=0.6292$, and $g_{4}=1$ [5], and the fractional bandwidth is set to $0.5 \%$. For this example, the filter consists of three half-wave superconducting transmission line resonators whose characteristic impedance is $50 \Omega$. From these values, we can derive the impedance matrix (18) by using [5].

In this case, all resonators have identical geometry and, thus, identical circuit distributed parameters $L_{0}, R_{0}, C_{0}$, and $G_{0}$. We also assume that the distributed parameters are constant through the whole resonator, as occurs in a straight transmission line. The cross section of the resonator is a microstrip geometry with $500-\mu \mathrm{m}$ linewidth, $400-\mathrm{nm}$ thickness of $\mathrm{YBaCuO}$ (YBCO), and $0.5-\mathrm{mm}$ thickness of $\mathrm{MgO}$ substrate. The surface resistance and penetration depth at $77 \mathrm{~K}$ are $0.7 \mathrm{~m} \Omega$ (at $10 \mathrm{GHz}$ ) and $230 \mathrm{~nm}$, respectively. The linear distributed parameters are obtained by using a finite-element electromagnetic simulator: $L_{0}=397 \mathrm{nH} / \mathrm{m}, R_{0}=0.153 \Omega / \mathrm{m}$, $C_{0}=158 \mathrm{pF} / \mathrm{m}, G_{0}=6.25 \mathrm{mS} / \mathrm{m}$. We assume a quadratic nonlinear dependence in (1) and (2), which, for a scaling critical current of $j_{2}=3 e 12 \mathrm{~A} / \mathrm{m}^{2}[19]$, results in nonlinear terms $\Delta L_{2}=7.74 \mathrm{pH} / \mathrm{mA}^{2}$ and $\Delta R_{2}=0.93 \mathrm{~m} \Omega / \mathrm{mA}^{2}$.

From these parameters, we apply (18)-(26) and find the current of the IMD in each resonator and the IMD transferred to the load. To do that, we consider two fundamental tones placed in the middle of the filter passband with $-10-\mathrm{dBm}$ input power. Table I details the magnitude and the relative phase of IMD to the fundamental signals of the current coupled to the load by each resonator and also the total current. In this case, we see that the second resonator is the one that contributes the most to the IMD, when the filter is fed with two tones in the middle of the band. Therefore, a way to reduce the IMD of this filter would be to widen the line of the second resonator.

The results presented here have been verified with simulations using a circuit analysis simulator based on harmonic balance, showing agreement within $3 \%$ in the IMD currents.

\section{B. Measurements in an Eight-Pole QE Filter}

Details on the filter design, layout, frequency response, and nonlinear measurements of the eight-pole QE filter, dealt with here, are reported in [17] and [18]. The initial parameters defining the nodal matrix [17] were adjusted to fit its measured frequency response. Note that this is crucial to obtain the fundamental signals at each resonator (Section III-A) and the transferred IMD to the load (Sections III-B and C). By using the adjusted design parameters, we repeat the procedure above

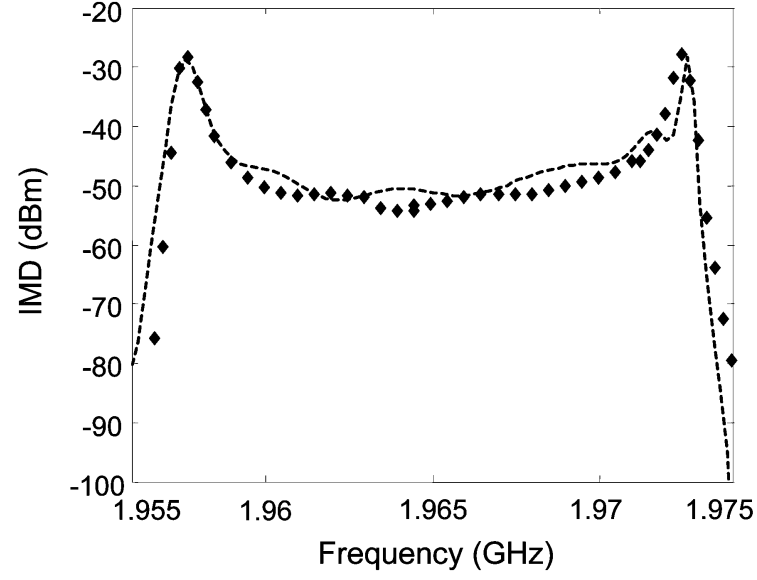

Fig. 4. Measured (diamonds) and adjusted results (dashed line) from the procedure described in Section III for IMD measurements in an eight-pole QE filter with two inputs tones $100 \mathrm{kHz}$ apart and an input power of $9.5 \mathrm{dBm}$. Details on these experiments may be found in [17] and [18].

(Section IV-A) for the eight-pole topology. Now the nonlinear distributed parameters are adjusted to fit the measured IMD [17]. Fig. 4 shows the measured (diamonds) and fitted (dashed line) using (25). These measurements consisted of measuring the IMD in the filter when the two fundamental tones are swept through the whole band of the filter. The two fundamental tones are $100 \mathrm{kHz}$ and their power is kept constant at $9.5 \mathrm{dBm}$.

Small discrepancies between the measurements and extracted IMD at some frequencies are due to small discrepancies between the in-band ripple of the measured filter frequency response and the one obtained from the nodal matrix. In spite of these, we observe good agreement in the whole frequency range. Cross-checking these results with harmonic-balance simulations have also shown very good agreement.

\section{ApPlication: Pre-Distortion, POSt-Distortion INTER-COMPENSATION FERROELECTRIC STAGES}

The possibility of compensating the inherent nonlinear response occurring in superconducting devices (such as transmission lines, resonators, and filters) by combination of different types of nonlinear effects has been discussed thus far. Here, we show three different ways of combining HTS and ferroelectric segments to reduce the nonlinear response in HTS bandpass filters. Outlines of these examples are shown in Fig. 5. We first propose a pre-distortion stage by using a ferroelectric transmission line as an input feeding line of the filter [see Fig. 5(a)]. Its complementary solution uses a ferroelectric line at the output of the filter as a post-distortion stage [see Fig. 5(b)]. Finally, in Fig. 5(c), we also propose a topology with intermediate ferroelectric stages to compensate the nonlinear effects of the superconductor, using the structure described in Fig. 1(e) with the restrictions discussed in Section II-B.3.

Below, we have determined the conditions for the cancellation of the nonlinear effects in these different approaches and assess their effects on the three-pole HTS Chebyshev filter topology presented in Section IV-A. Fig. 6 shows the fundamental signal (thin solid line) and IMD signals (dashed line) at the output of the filter when two feeding tones, at 


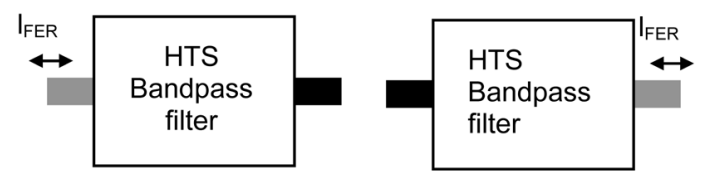

a)

b)

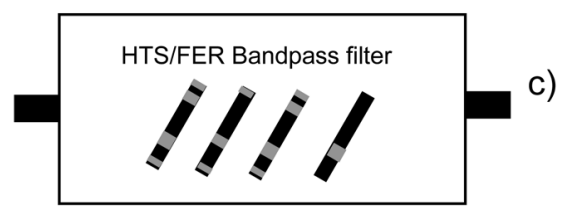

Fig. 5. (a) Ferroelectric segment for nonlinear pre-distortion in an HTS bandpass filter. (b) Ferroelectric segment for nonlinear post-distortion in an HTS bandpass filter. (c) Intermediate ferroelectric compensation stages.

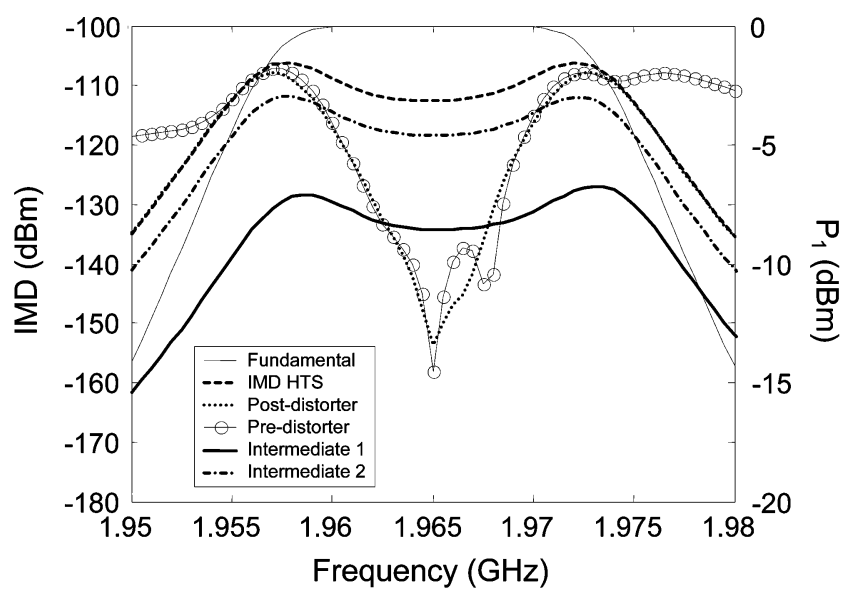

Fig. 6. Simulated response of the filter. Thin solid line represents the fundamental output power P1. Dashed line: IMD for an HTS filter. Dots and circles represent the IMD for an HTS filter with post- and pre-distortion ferroelectric stages, respectively. Dotted-dashed and thick solid lines represent the IMD for an HTS filter with intermediated ferroelectric stages.

$0 \mathrm{dBm}$, are swept through the bandpass of the filter. Note that the fundamental at the output of the filter defines the transfer coefficient of the filter $\left(S_{21}\right)$.

\section{A. Ferroelectric Transmission Line Pre-Distorter}

By using the expressions of Section II for the nonlinear effects in the ferroelectric transmission line and the expression of Section III for the nonlinear effects in an HTS filter, we obtain the condition such that the pre-distorter ferroelectric line cancels the IMD occurring in the HTS filter as

$$
\begin{aligned}
& \frac{3}{2} e^{-2 \alpha_{F} l_{F}} \sum_{j=1}^{N} Z_{i 0}\left|Z_{i 0}\right|^{2} A_{H, 12}\left(\sqrt{\frac{8 Q_{0 i}}{\omega R_{i} L_{d_{i}} l_{i}}}\right)^{3 / 2} \frac{Q_{L j}}{\omega_{12} L_{d i}} k_{L j} \\
& =-Z_{0}\left|Z_{0}\right|^{2} A_{F, 12} l_{F} Z_{N+1,1} \text {. }
\end{aligned}
$$

Note that the term accounting for the nonlinear effects occurring on the ferroelectric transmission line at the input of the filter should be scaled by the transfer coefficient of the filter $Z_{N+1,1}$, whereas the terms accounting for the nonlinear effects in the HTS filter are affected by the propagation of the fundamental signals through the ferroelectric input feeding line.
As occurring for the pre-distorter case in a single resonator, the pre-distortion may be adjusted by tuning the length $l_{F}$ of the feeding input line, without altering the filter performance.

Fig. 6 shows (in circles) the IMD at the output of the filter when the length $l_{F}$ of the ferroelectric line has been adjusted to cancel the IMD in the middle of the filter band. While strong suppression occurs in the middle of the band, the nonlinear response at the edges of the bands is not compensated. Moreover, due to the lack of frequency selection of the ferroelectric line, high IMD appears out of the filter band.

\section{B. Ferroelectric Transmission Line Post-Distorter}

In analogy with the previous case, we may find the compensation condition using an output ferroelectric transmission line as a post-distorter stage as

$$
\begin{array}{r}
\frac{3}{2} \sum_{j=1}^{N} Z_{i 0}\left|Z_{i 0}\right|^{2} A_{H, 12}\left(\sqrt{\frac{8 Q_{0 i}}{\omega R_{i} L_{d_{i}} l_{i}}}\right)^{3 / 2} \frac{Q_{L j}}{\omega_{12} L_{d i}} k_{L j} \\
=-Z_{0}\left|Z_{0}\right|^{2} A_{F, 12} l_{F} .
\end{array}
$$

Again the length $l_{F}$ of the ferroelectric line will allow us to control the compensation effect. The results are shown via the dotted line in Fig. 6. As in the pre-distorter case, strong cancellation occurs in the middle of the filter band and no cancellation is observed at the edges of the band. However, in contrast to the pre-distorter case, the IMD at the output band of the filter is not increasing.

In practice, most of HTS bandpass filter consists of high-order filters where the IMD remains almost flat in the bandpass and only sharply peaks at the edges of the filter [15], making these two previous simple approaches very useful in real applications. However, to ensure cancellation of the nonlinear effects through the whole band of the filter and for any kind of incident signals, the combined nonlinear distributed effects should follow identical frequency dependence. To overcome this, the next approaches include inter-compensation stages along the filter.

\section{Inter-Compensation Ferroelectric Stages}

The compensation condition for this case, shown in Fig. 5(c), where two types of nonlinear effects are distributed along the filter structure, may be obtained from (25) as

$$
\begin{aligned}
& \sum_{j=1}^{N} T_{j} \gamma_{j} I_{j_{-} f_{1}}^{2} I_{j_{-} f_{2}}^{*} A_{H, 12} \Gamma_{H}^{\prime} \frac{Q_{L j} k_{L j}}{\omega W_{0 j}} \\
& \quad=-\sum_{j=1}^{N} T_{j} \gamma_{j} I_{j_{-} f_{1}}^{2} I_{j_{-} f_{2}}^{*} Z_{i 0}\left|Z_{i 0}\right|^{2} A_{F, 12} \Gamma_{F}^{\prime} \frac{Q_{L j} k_{L j}}{\omega W_{0 j}} .
\end{aligned}
$$

Once the design of the filter is decided, the adjustable parameters for the cancellation are the geometric factors $\Gamma_{F}^{\prime}$ and $\Gamma_{H}^{\prime}$, defined in Section II-B. These parameters depend on the distribution and length of the segments with ferroelectric and superconducting nonlinear effects along the filter structure. Therefore, there are many combinations of segments with different types of nonlinear effects that can be used to reduce the nonlinear response of the filter. 
The theoretical complete cancellation of IMD across the filter passband suggested by (29) has been verified through numerical simulations using harmonic balance. Additionally, and as an example, we have assessed a case where the three half-wave resonators in the filters have an identical distribution of HTS and dielectric nonlinearities. These resonators consisted of ferroelectric segments at the edges of the half-wave line and an HTS segment in the center of the line. For a superconductive nonlinear term $A_{H, 12}$, obtained from the above example (Section IV-A) and ferroelectric nonlinear terms $A_{F, 12}$, obtained from preliminary measurements on coplanar waveguide (CPW) transmission lines [9], Fig. 6 shows the reduction in the nonlinear response when the length of the HTS segment is $8 \lambda / 20$ (dotted-dashed line) and $9 \lambda / 20$ (thick solid line). These show how the cancellation can be adjusted by tuning the geometric factors $\Gamma_{H}^{\prime}$ and $\Gamma_{F}^{\prime}$. In these two examples, we see how the reduction on the nonlinear responses remains constant through the whole frequency band of the filter.

\section{DISCUSSION AND CONCLUSION}

We have discussed the theoretical feasibility of suppressing the IMD generated by HTS materials with the use of a nonlinear dielectric (possibly a ferroelectric). The configurations explored avoid having to use a ferroelectric in the whole HTS planar layout, thus avoiding losses that the ferroelectric would produce.

Ferroelectric loss has been a limiting issue in the development of tunable HTS filters [20]. We think this might not be the case in the use of ferroelectrics for IMD compensation [21]. We base this on the fact that, to produce a useful shift in the resonant frequency, the interaction of a ferroelectric with the RF fields in a resonator has to be much stronger than that required to produce a weak IMD signal [21]. Thus, as discussed in [21], we hope that a significant reduction of the IMD in superconducting filters can be achieved with the use of ferroelectrics without degrading the quality factors in their resonators to the point where the selectivity or the insertion loss is severely affected. In any case, the theoretical work in this paper should be useful to efficiently deploy the ferroelectric for maximum IMD reduction with a minimum effect on losses.

\section{REFERENCES}

[1] J.-S. Hong, M. J. Lancaster, D. Jedamzik, and R. B. Greed, "On the development of superconducting microstrip filters for mobile communications applications," IEEE Trans. Microw. Theory Tech., vol. 47, no. 9, pp. 1656-1663, Sep. 1999.

[2] B. A. Willemsen, , H. Weinstock and M. Nisenoff, Eds., "HTS wireless applications," in Microwave Superconductivity, ser. NATO Sci. E: Appl. Sci. Norwell, MA: Kluwer, 2002, vol. 375, ch. 15, pp. 387-416.

[3] B. A. Willemsen, B. H. King, T. Dahm, and D. J. Scalapino, "Microwave intermodulation in high- $T c$ superconducting microstrip resonators," IEEE Trans. Appl. Supercond., vol. 9, no. 2, pp. 4181-4184, Jun. 1999.

[4] D. Seron, C. Collado, J. Mateu, and J. M. O'Callaghan, "Analysis and simulation of distributed nonlinearities in ferroelectrics and superconductors for microwave applications," IEEE Trans. Microw. Theory Tech., vol. 54, no. 3, pp. 1154-1160, Mar. 2006.

[5] J.-S. Hong and M. J. Lancaster, Microstrip Filters for RF/Microwave Applications, ser. Microw. Opt. Eng.. $\quad$ New York: Wiley, 2001.
[6] C. Collado, J. Mateu, and J. M. O'Callaghan, "Analysis and simulation of the effects of distributed nonlinearities in microwave superconducting devices," IEEE Trans. Appl. Supercond., vol. 15, no. 1, pp. 26-29, Mar. 2005.

[7] J. Mateu, J. C. Booth, and S. A. Schima, "Characterization of the nonlinear response in ferroelectric thin-film transmission lines," IEEE Trans. Microw. Theory Tech., submitted for publication.

[8] J. C. Booth, R. H. Ono, I. Takeuchi, and K.-S. Chang, "Microwave frequency tuning and harmonic generation in ferroelectric thin film transmission lines," Appl. Phys. Lett., vol. 81, no. 4, pp. 718-720, 2002.

[9] J. Mateu, J. C. Booth, and S. A. Schima, "Frequency tuning and spurious signal generation at microwave frequencies in ferroelectric $\mathrm{SrTiO}_{3}$ thin-film transmission lines," IEEE Trans. Microw. Theory Tech., vol. 55, no. 2, pp. 391-396, Feb. 2007.

[10] J. C. Booth, L. R. Vale, and R. H. Ono, "On-wafer measurements of nonlinear effects in high-temperature superconductors," IEEE Trans. Appl. Supercond., vol. 11, no. 1, pp. 1387-1391, Mar. 2001.

[11] D. E. Oates, S. H. Park, D. Agassi, G. Koren, and K. Irgmaier, "Temperature dependence of intermodulation distortion in YBCO: Understanding nonlinearity," IEEE Trans. Appl. Supercond., vol. 15, no. 2, pp. 3308-3312, Jun. 2005.

[12] J. Mateu, C. Collado, O. Menendez, and J. M. O'Callaghan, "Experiments and model of intermodulation distortion in a rutile resonator with YBaCuO," J. Supercond., vol. 16, no. 5, pp. 873-880, 2003.

[13] J. C. Booth, K. Leong, S. A. Schima, A. Jargon, D. C. Degroot, and R. Schwall, "Phase-sensitive measurements of nonlinearity in high-temperature superconductor thin film," IEEE Trans. Appl. Supercond., vol. 15, no. 2, pp. 1000-1003, Jun. 2005.

[14] M. J. Lancaster, Passive Microwave Devices Applications of High Temperature Superconductors. Cambridge, U.K.: Cambridge Univ. Press, 1997.

[15] M. I. Salkola, "Intermodulation response of superconducting filters," $J$. Appl. Phys., vol. 98, Jul. 2005, 023907.

[16] D. Pozar, Microwave Engineering. New York: Wiley, 1998.

[17] J. Mateu, C. Collado, O. Menendez, and J. M. O'Callaghan, "Nonlinear performance characterization in an eight-pole quasi-elliptic band filter," Supercond. Sci. Technol., vol. 17, pp. S359-S362, 2004.

[18] C. Collado, J. Mateu, O. Menendez, and J. M. O'Callaghan, "Nonlinear distortion in an eight-pole quasi-elliptic bandpass HTS filter for CDMA system," IEEE Trans. Appl. Supercond., vol. 15, no. 2, pp. 992-995, Jun. 2005.

[19] K. Leong, J. C. Booth, and S. A. Schima, "A current-density scale for characterizing nonlinearity in high-Tc superconductors," IEEE Trans. Appl. Supercond., vol. 15, no. 2, pp. 3608-3611, Jun. 2005.

[20] B. H. Moeckly and Y. Zhang, "Strontium titanate thin films for tunable YbaCuO microwave filters," IEEE Trans. Appl. Supercond., vol. 11, no. 1, pp. 450-453, Mar. 2001

[21] J. Mateu, J. C. Booth, and B. H. Moeckly, "Nonlinear response of combined superconductor/ferroelectric devices: First experimental step," IEEE Trans. Appl. Supercond., to be published.

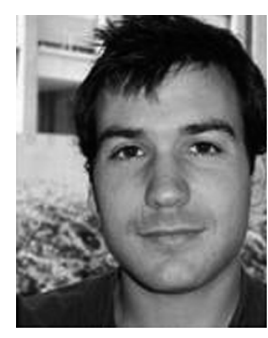

Jordi Mateu (M'03) was born in Llardecans, Spain, in 1975. He received the Telecommunication Engineering and $\mathrm{Ph} . \mathrm{D}$. degrees from the Universitat Politècnica de Catalunya (UPC), Barcelona, Spain, in 1999 and 2003, respectively.

Since October 2006, he has been Research Fellow with the Department of Signal Theory and Communications, UPC. From May to August 2001, he was Visiting Researcher with Superconductor Technologies Inc., Santa Barbara, CA. From October 2002 to August 2005, he was Research Associate with the Telecommunication Technological Center of Catalonia, Catalonia, Spain. Since September 2004, he has held several Guest Researcher appointments with the National Institute of Standards an Technology (NIST), Boulder, CO, where he was a Fulbright Research Fellow from September 2005 to October 2006. In July 2006, he was a Visiting Researcher with the Massachusetts Institute of Technology (MIT) Lincoln Laboratory. From September 2003 to August 2005, he was a Part-Time Assistant Professor with the Universitat Autònoma de Barcelona. His primary research interests include microwave devices and system and characterization and modeling of new electronic materials including ferroelectrics, magnetoelectric, and superconductors.

Dr. Mateu was the recipient of the 2004 Prize for the best doctoral thesis in fundamental and basic technologies for information and communications presented by the Colegio Oficial Ingenieros de Telecomunicación (COIT) and the Asociación Española de Ingenieros de Telecomunicación (AEIT). He was also 
the recipient of a Fulbright Research Fellowship, an Occasional Lecturer Award for visiting MIT, and a Ramón y Cajal Contract.

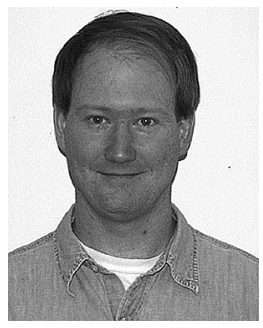

James C. Booth received the B.A. degree in physics from the University of Virginia, Blacksburg, in 1989, and the Ph.D. degree in physics from the University of Maryland at College Park, in 1996. His doctoral dissertation concerned novel measurements of the frequency-dependent microwave surface impedance of cuprate thin-film superconductors.

Since 1996, he has been a Physicist with the National Institute of Standards and Technology (NIST), Boulder, CO, originally as a National Research Council (NRC) Post-Doctoral Research Associate (1996-1998) and currently as a Staff Scientist. His research with NIST is focused on exploring the microwave properties of new electronic materials and devices including ferroelectric, magneto-electric, and superconducting thin films, as well as developing experimental platforms integrating microfluidic and microelectronic components for RF and microwave frequency characterization of liquid and biological samples.

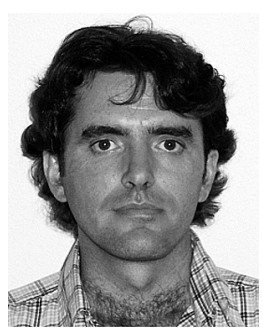

Carlos Collado (M'05) was born in Barcelona, Spain, in 1969. He received the Telecommunication Engineering sand $\mathrm{Ph} . \mathrm{D}$. degrees from the Technical University of Catalonia (UPC), Barcelona, Spain, in 1995 and 2001, respectively.

In 1998, he joined the faculty of UPC, where he has been teaching courses on the theory of electromagnetism, microwave laboratory, and high-frequency devices and systems. In 2004, he was a Visiting Researcher with the University of California at Irvine. Since April 2005, he has been an Associate Professor with UPC. His primary research interests include microwave devices and systems, electrooptics applications, and superconducting devices.

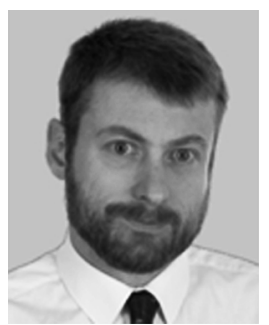

Juan M. O'Callaghan (SM'01) received the Telecommunication Engineering degree from the Universitat Politècnica de Catalunya (UPC), Barcelona, Spain, in 1987, and the M.S. and Ph.D. degrees from the University of Wisconsin-Madison, in 1989 and 1992, respectively.

He is currently a Full Professor with UPC. He was an intern with the Systems Research Center, Honeywell, Bloomington, $\mathrm{MN}$, where he was involved with noise measurement methods for FETs at $K a$-band. From 2003 to 2006 he was Manager for MERIT, a consortium of European universities delivering a joint master's program in information technologies within the Erasmus Mundus Program. He is currently Vice-Dean of Academic Affaires with Telecom BCN, the telecommunication engineering school of UPC. He has authored or coauthored over 40 papers in peer-reviewed international magazines. He holds three patents. His research interests include microwave devices and materials and microwave photonics. $\mathrm{He}$ has been involved with noise characterization, large-signal properties of GaAs FETs, and advanced microwave materials such as superconductors and ferroelectrics. 